\title{
Presymplectic AKSZ formulation of Einstein gravity
}

\author{
Maxim Grigoriev ${ }^{a, b}$ and Alexei Kotov ${ }^{c}$ \\ ${ }^{a}$ Lebedev Institute of Physics, \\ Leninsky ave. 53, 119991 Moscow, Russia \\ ${ }^{b}$ Institute for Theoretical and Mathematical Physics, \\ Lomonosov Moscow State University, 119991 Moscow, Russia \\ ${ }^{c}$ Faculty of Science, University of Hradec Kralove, \\ Rokitanskeho 62, Hradec Kralove 50003, Czech Republic \\ E-mail: grig@lpi.ru, oleksii.kotov@uhk.cz
}

ABSTRACT: Any local gauge theory can be represented as an AKSZ sigma model (upon parameterization if necessary). However, for non-topological models in dimension higher than 1 the target space is necessarily infinite-dimensional. The interesting alternative known for some time is to allow for degenerate presymplectic structure in the target space. This leads to a very concise AKSZ-like representation for frame-like Lagrangians of gauge systems. In this work we concentrate on Einstein gravity and show that not only the Lagrangian but also the full-scale Batalin-Vilkovisky (BV) formulation is naturally encoded in the presymplectic AKSZ formulation, giving an elegant supergeometrical construction of BV for Cartan-Weyl action. The same applies to the main structures of the respective Hamiltonian BFV formulation.

Keywords: BRST Quantization, Classical Theories of Gravity, Differential and Algebraic Geometry, Gauge Symmetry

ArXIV EPRINT: 2008.11690 


\section{Contents}

1 Introduction 1

2 Presymplectic AKSZ form of Cartan-Weyl action 3

3 BV-AKSZ interpretation of the model $\quad 4$

3.1 The structure of the fiber and its symplectic quotient 6

$\begin{array}{lll}3.2 & \text { BV from presymplectic AKSZ } & 10\end{array}$

4 The origin of the target space structures 13

5 BFV phase space from presymplectic AKSZ 15

6 Conclusions 17

$\begin{array}{ll}\text { A The structure of the kernel } & 18\end{array}$

\section{Introduction}

Batalin-Vilkovisky (BV) formalism [1,2] has proved a powerful tool not only in quantization but more generally in analyzing physical content of gauge systems and even constructing new gauge models. In the context of local gauge theories the appropriate enhancement [35] (see also [6-8] for earlier important developments) of the BV approach operates in terms of the jet-bundles associated to fields, ghost fields and antifields.

If one is only interested in the equations of motion and hence disregards Lagrangians and associated graded symplectic structures the appropriate version [9] (see also [10, 11]) of the local BV formalism can be formulated immediately in terms of manifolds which are more general than jet-bundles, giving a powerful generalization [12, 13] of the standard approach and leading to more invariant and flexible description of gauge systems.

More precisely, a generic local gauge field theory formulated at the level of equations of motion can be represented [12] as a nonlagrangian version [14] of AKSZ-type sigma model, whose target space is a BV jet-bundle of the system or one of its equivalent reductions. In this way one can define and analyze local gauge field theories in terms of generic $Q$ manifolds that are not necessarily jet-bundles. This approach can be regarded as a BV extension of the invariant geometrical approach to PDEs [15] (see also [16, 17]). It can also be considered as an extension of the AKSZ construction [18] to the case of not necessarily topological gauge theories.

At the Lagrangian level a generic local gauge system can be also represented [19, 20] as an AKSZ-type sigma model whose target space is a suitably defined graded cotangent bundle over the jet-bundle associated to fields and ghosts or one of its equivalent reductions. 
This approach also known as the Lagrangian parent formulation has certain remarkable features. In particular, just like conventional AKSZ sigma models it automatically contains Batalin-Fradkin-Vilkovisky (BFV) [21, 22] Hamiltonian formulation. Moreover, the approach gives a systematic way to derive frame-like description of the system: for instance in the case of Einstein gravity the familiar frame-like (also known as Cartan-Weyl) formulation [23] in terms of the frame-field and Lorentz connection can be systematically arrived at [19] as a suitable equivalent reduction of the parent formulation for the metric-like formulation of Einstein gravity.

Despite its nice supergeometrical structure, the Lagrangian parent formulation involves an infinite tower of generalized auxiliary fields. It turns out that by eliminating most of auxiliaries but at the same time trying to keep the supergeometrical structure intact one can naturally arrive at so-called presymplectic AKSZ formulations [24]. These have the form of finite-dimensional AKSZ sigma models whose target space presymplectic structure is allowed to be degenerate. In this way one can find elegant presymplectic AKSZ formulations [24] for a variety of gauge theories including the frame-like form of Einstein gravity. It turns out that the ghost-independent part of the presymplectic AKSZ action for gravity is precisely the Cartan-Weyl action. However, if space-time dimension is greater than 3 the BV-like 2-form defined on the space of supermaps to the target space is degenerate and the BV interpretation of such a presymplectic AKSZ sigma model has remained somewhat unclear.

Later on it was realised [25] that the presymplectic 2-form on the target space is closely related to a BV extension of the canonical 2-form on the stationary surface, which is induced by the Lagrangian. As we are going to see it can also be seen as a BV symplectic structure completed to a cocycle of the total differential $\mathrm{d}_{\mathrm{h}}+s$ and transferred to the minimal formulation of the BV-BRST complex. ${ }^{1}$ Here, $\mathrm{d}_{\mathrm{h}}$ denotes the horizontal differential and $s$ the BV-BRST differential of the theory.

In this work we concentrate on the example of Einstein Gravity and give a consistent interpretation of its presymplectic AKSZ formulation. More specifically, we demonstrate that the BV presymplectic 2-form on the space of supermaps is regular and factoring out its kernel results in the standard symplectic BV field-antifield configuration space while the AKSZ action functional induces the BV master action, giving a concise and geometrical BV formulation of the frame-like gravity. Analogous procedure applied to the presymplectic AKSZ sigma model restricted to the spatial slice of the space-time results in the BFV phase space and 1-st class constraints of the frame-like gravity. However the presymplectic structure in this case is not regular and the phase space is recovered as a maximal symplectic submanifold of the respective space of supermaps. Up to this subtlety, just like in the case of usual AKSZ, its presymplectic version also contains both BV and $\mathrm{BFV}$ formulations. Note that the BFV phase-space encoded in the proposed presymplectic formulation is precisely the one of $[27,28]$ (see also [29]), where the relation between BV and $\mathrm{BFV}$ for the frame-like gravity has been recently studied.

\footnotetext{
${ }^{1}$ The descent symplectic structures completing the BV symplectic structure have been discussed in [26].
} 


\section{Presymplectic AKSZ form of Cartan-Weyl action}

Let $(V, \eta)$ be a Minkowski space $V$ of $n$-dimensions. In the basis $e_{a}$ the metric coefficients are $\eta_{a b}=\eta\left(e_{a}, e_{b}\right)$. The algebra of its symmetries is Poincaré algebra which is a semidirect sum of Lorentz subalgebra $o(n-1,1)$ and the abelian sublagebra of translations. It is useful to identify translations with $V$ itself and Lorentz subalgebra as $V \wedge V$. This gives a natural basis $T_{a}=e_{a}, L_{b c}=e_{b} \wedge e_{c}$ in the Poincare algebra. In what follows we consider a more general Lie algebra structure on the same linear space, namely, (A)dS or Poincare algebra structure. In the above basis the commutation relations read as:

$$
\begin{aligned}
{\left[T_{a}, T_{b}\right] } & =\lambda L_{a b}, \quad\left[L_{a b}, T_{c}\right]=\eta_{b c} T_{a}-\eta_{a c} T_{b}, \\
{\left[L_{a b}, L_{c d}\right] } & =L_{a d} \eta_{b c}-L_{b d} \eta_{a c}-L_{a c} \eta_{b d}+L_{b c} \eta_{a d},
\end{aligned}
$$

where $\lambda \in \mathbb{R}$ is related to the cosmological constant through $\lambda=-\frac{2 \Lambda}{(n-1)(n-2))}$ so that at $\lambda=0$ the above relations become those of the Poincaré algebra.

Now we switch to the language of graded geometry and consider the associate graded manifold $\mathfrak{g}[1]$ which is the above Lie algebra $\mathfrak{g}$ seen as a linear space and with the degree of linear coordinates shifted by 1 . The Lie algebra structure on $\mathfrak{g}$ defines a $Q$-structure on $\mathfrak{g}[1]$, which can be identified with the Chevalley-Eilenberg $(\mathrm{CE})$ differential of $\mathfrak{g}$. In terms of the coordinates it is given by

$$
q \xi^{a}=\rho_{b}^{a} \xi^{b}, \quad q \rho^{a b}=\rho^{a}{ }_{c} \rho^{c b}+\lambda \xi^{a} \xi^{b}
$$

where $\rho^{a}{ }_{c}=\rho^{a b} \eta_{b c}$. A more invariant way to define $q$ is to introduce a canonical linear function $\Psi$ on $\mathfrak{g}[1]$ with values in $\mathfrak{g}$ itself. In terms of coordinates it is given by $\Psi=$ $\xi^{a} T_{a}+\frac{1}{2} \rho^{a b} L_{a b}$. Then $q$ can be defined through the relation

$$
q \Psi=\frac{1}{2}[\Psi, \Psi]
$$

where the action of $q$ on functions on $\mathfrak{g}[1]$ is extended naturally to functions with values in linear space $\mathfrak{g}$ while the Lie algebra structure on $\mathfrak{g}$ is extended to $\mathfrak{g}$-valued functions by linearity.

On $\mathfrak{g}[1]$ there is a natural $q$-invariant presymplectic structure of degree $n-1$, i.e. a closed 2-form of degree $n-1$, which reads as [24]:

$$
\boldsymbol{\omega}^{\mathfrak{g}[1]}=\mathcal{V}_{a b c}(\xi) d \xi^{a} d \rho^{b c}, \quad \mathcal{V}_{a_{1} \ldots a_{k}}(\xi)=\frac{1}{(n-k) !} \epsilon_{a_{1} \ldots a_{k} b_{1} \ldots b_{n-k}} \xi^{b_{1}} \ldots \xi^{b_{n-k}}
$$

It follows from $L_{q} \boldsymbol{\omega}^{\mathfrak{g}[1]}=0$ and $d \boldsymbol{\omega}^{\mathfrak{g}[1]}=0$ that $d \mathfrak{i}_{q} \boldsymbol{\omega}^{\mathfrak{g}[1]}=0$ and hence

$$
\mathfrak{i}_{q} \boldsymbol{\omega}^{\mathfrak{g}[1]}=d \mathcal{H}, \quad \mathfrak{i}_{q} \mathfrak{i}_{q} \boldsymbol{\omega}^{\mathfrak{g}[1]}=0=q \mathcal{H},
$$

for some function $\mathcal{H}$ of degree $n$. Furthermore, there exists a presymplectic potential $\chi \in \Lambda^{1}(\mathfrak{g}[1])$ such that $\boldsymbol{\omega}^{\mathfrak{g}[1]}=d \chi$. In the case at hand one can take:

$$
\chi=\mathcal{V}_{a b}(\xi) d \rho^{a b}, \quad \mathcal{H}=\mathcal{V}_{a b}(\xi)\left(\rho^{a}{ }_{c} \rho^{c b}+\frac{(n-2) \lambda}{n} \xi^{a} \xi^{b}\right)
$$


Note that in contrast to $\chi$, which is defined up to a $d$-closed 1 -form, function $\mathcal{H}$ is uniquely determined by $\boldsymbol{\omega}^{\mathfrak{g}[1]}$ and $q$. It is important to stress that $\boldsymbol{\omega}^{\mathfrak{g}[1]}$ is not a regular presymplectic form for $n>3$ as it vanishes at $\xi^{a}=0$.

Let us consider the presymplectic AKSZ sigma model with the source space being $\left(T[1] X, d_{X}\right)$, where $X$ is a space-time manifold of dimension $n>2$ and $d_{X}$ is the de Rham differential seen as a homological vector field on $T[1] X$, and the target space being $\left(\mathfrak{g}[1], q, \boldsymbol{\omega}^{\mathfrak{g}[1]}\right)$. Recall that $T[1] X$ denotes a graded manifold obtained from $T X$ via shifting by one the degree of fiber coordinates; the algebra of functions on $T[1] X$ is the graded algebra of differential forms on $X$. Maps from $\left(T[1] X, d_{X}\right)$ to $\left(\mathfrak{g}[1], q, \boldsymbol{\omega}^{\mathfrak{g}[1]}\right)$ are field configurations of the Cartan-Weyl formulation of gravity. Indeed, in terms of coordinates a map $\sigma$ is parameterized by

$$
\sigma^{*}\left(\xi^{a}\right)=e_{\mu}^{a}(x) \theta^{\mu}, \quad \sigma^{*}\left(\rho^{a b}\right)=\omega_{\mu}^{a b}(x) \theta^{\mu},
$$

where $x^{\mu}, \theta^{\mu}$ are standard local coordinates on $T[1] X$ induced by local coordinates on the base $X$ and $\sigma^{*}$ is a pullback map associated to $\sigma$. Functions $e_{\mu}^{a}(x), \omega_{\mu}^{a b}(x)$ can be taken as coordinates on the space of maps and are naturally identified with the components of the frame field and Lorentz connection. In view of this identification it is natural to restrict to maps such that $e_{\mu}^{a}(x)$ is invertible.

The data of AKSZ sigma-model determine the action functional as follows:

$$
S[\sigma]=\int_{T[1] X}\left(\sigma^{*}(\chi)\left(d_{X}\right)+\sigma^{*}(\mathcal{H})\right)
$$

where $\chi \in \bigwedge^{1}(\mathfrak{g}[1])$ and $\mathcal{H} \in \bigwedge^{0}(\mathfrak{g}[1])$ are given in (2.6). In component one has:

$$
\begin{aligned}
S[e, \omega] & =\int_{X} \mathcal{V}_{a b}(e)\left(d_{X} \omega^{a b}+\omega^{a}{ }_{c} \omega^{c b}+\frac{(n-2) \lambda}{n} e^{a} e^{b}\right) \\
& =\int_{X} \mathcal{V}_{a b}(e)\left(d_{X} \omega^{a b}+\omega^{a}{ }_{c} \omega^{c b}\right)-2 \Lambda \mathcal{V}(e)
\end{aligned}
$$

where fields $e^{a}, \omega^{a b}$ parameterize $\sigma$ according to (2.7) and the wedge product of space-time differential forms is assumed. This is indeed a familiar Cartan-Weyl action.

\section{BV-AKSZ interpretation of the model}

If $\boldsymbol{\omega}^{\mathfrak{g}[1]}$ is nondegenerate (which is not the case for gravity in $n>3$ ) the BV formulation is extracted from the AKSZ data as follows: the BV field-antifield space is the space of supermaps from $T[1] X$ to the target supermanifold. In contrast to the space of maps the space of supermaps is a graded manifold. The coordinates there can be introduced as follows

$$
\begin{aligned}
\widehat{\sigma}^{*}\left(\xi^{a}\right) & =\stackrel{0}{\xi}^{a}(x)+e_{\mu}^{a}(x) \theta^{\mu}+\frac{1}{2} \stackrel{\xi}{\xi}_{\mu \nu}^{a}(x) \theta^{\mu} \theta^{\nu}+\ldots, \\
\widehat{\sigma}^{*}\left(\rho^{a b}\right) & ={ }^{0} \rho^{a b}(x)+\omega_{\mu}^{a b}(x) \theta^{\mu}+\frac{1}{2}{ }^{2} \rho_{\mu \nu}^{a b}(x) \theta^{\mu} \theta^{\nu}+\ldots
\end{aligned}
$$


where now the form-degree $k$ components carry ghost degree $1-k$. The space of maps is recovered by setting to zero all the coordinates of nonvanishing degree. In other words, the space of maps is a body of the space of supermaps. Moreover, the space of supermaps is naturally a bundle over the respective space of maps.

The target space symplectic structure $\boldsymbol{\omega}^{\mathfrak{g}[1]}$ determines a BV symplectic structure of degree -1 on the space of supermaps:

$$
\boldsymbol{\omega}^{\mathrm{BV}}=\int_{T[1] X} \widehat{\sigma}^{*}\left(\boldsymbol{\omega}_{A B}^{\mathfrak{g}[1]}\right) \delta \psi^{A}(x, \theta) \wedge \delta \psi^{B}(x, \theta),
$$

where we introduced a collective notation $\psi^{A}$ for target space coordinates $\xi^{a}, \rho^{a b}$ and $\psi^{A}(x, \theta)=\widehat{\sigma}^{*}\left(\psi^{A}\right)$. It is nondegenerate provided $\boldsymbol{\omega}^{\mathfrak{g}[1]}$ is. The $\mathrm{BV}$ action is given by

$$
S^{\mathrm{BV}}[\widehat{\sigma}]=\int_{T[1] X}\left(\widehat{\sigma}^{*}(\chi)\left(d_{X}\right)+\widehat{\sigma}^{*}(\mathcal{H})\right),
$$

where the difference with (2.8) is in $\sigma$ replaced by $\widehat{\sigma}$. In particular, setting fields of nonzero degree to zero one recovers (2.8). For further details and developments of the AKSZ approach we refer to $[11,14,18,20,30-41]$.

In the presymplectic case one can still define the BV-like action (3.4) and the presymplectic structure (3.3) on the space of supermaps. Just like in the usual AKSZ approach the BV-like action has the same structure as the classical action and in our case can be written explicitly as:

$$
S^{\mathrm{BV}}[\widehat{\xi}, \widehat{\rho}]=\int_{T[1] X} \mathcal{V}_{a b}(\widehat{\xi})\left(d_{X} \widehat{\rho}^{a b}+\widehat{\rho}^{a}{ }_{c} \widehat{\rho}^{c b}+\frac{(n-2) \lambda}{n} \widehat{\xi}^{a} \widehat{\xi}^{b}\right)
$$

where $\widehat{\xi}^{a}(x, \theta)=\widehat{\sigma}^{*}\left(\xi^{a}\right)$ and $\widehat{\rho}^{a b}(x, \theta)=\widehat{\sigma}^{*}\left(\rho^{a b}\right)$. The master action satisfies an analog of the master equation that can be defined as follows: the homological vector field $q$ in the target space and the de Rham differential $d_{X}$ on $X$ naturally define the BRST differential $s$ on the space of supermaps [18]:

$$
s=\int d^{n} x d^{n} \theta\left(d_{X} \psi^{A}(x, \theta)+q^{A}(\psi(x, \theta)) \frac{\delta}{\delta \psi^{A}(x, \theta)} .\right.
$$

One can then check that by construction

$$
\boldsymbol{\omega}^{\mathrm{BV}}(s, s)=0 \quad \mathfrak{i}_{s} \boldsymbol{\omega}^{\mathrm{BV}}=\delta S^{\mathrm{BV}},
$$

modulo boundary terms.

As we are going to see the presymplectic structure is regular in a certain precise sense and hence it defines the symplectic structure on the symplectic quotient space, i.e. the space of leaves of the kernel distribution determined by $\boldsymbol{\omega}^{\mathrm{BV}}$. One can then check that $S^{\mathrm{BV}}$ is annihilated (modulo boundary terms) by the distribution and hence defines a well defined functional on the space of leaves. Moreover, in a similar way both $s$ and $\boldsymbol{\omega}^{\mathrm{BV}}$ induce the respective structures on the quotient and altogether they satisfy the analog of (3.7). Finally, because the presymplectic structure induced on the quotient is nondegenerate (3.7) 
implies usual BV master equation on the symplectic quotient and hence this data defines a conventional BV formulation on the symplectic quotient.

Furthermore, one can check that the kernel of $\omega^{\mathrm{BV}}$ is generated by vector fields of nonvanishing degree and hence the symplectic quotient is still a bundle over the space of maps. It follows the ghost-independent part of the BV action on the quotient is just the CartanWeyl action (2.9). Taking into account that the symplectic structure is nondegenerate on the quotient, the spectrum of ghost fields precisely corresponds to the gauge invariance of the action, and the master equation holds it follows that we have indeed arrived at the BV formulation of gravity in the Cartan-Weyl form.

Because the above considerations deal with infinite-dimensional manifolds some care is required. However, as we are going to see in the next section taking the symplectic quotient of the space of supermaps boils down to that of a finite-dimensional manifold while the construction of basic objects can be made precise by employing the jet-bundle technique.

Moreover, it turns out the symplectic quotient can be explicitly realised as a submanifold $J_{X}(N)$ of the entire manifold $J_{X}(M)$ of supermaps from $T[1] X$ to $\mathfrak{g}[1]$, which is transversal to the kernel distribution. In this way $S^{\mathrm{BV}}, \boldsymbol{\omega}^{\mathrm{BV}}$ on the quotient can be obtained by simply pulling back these structures to $J_{X}(N)$.

It is important to stress that in order to study the theory there is no need to explicitly identify the symplectic quotient. The master equation, gauge fixing etc. can be implemented just in terms of $J_{X}(M)$. Moreover, constraints determining the submanifold do not involve space-time derivatives so that they can be easily implemented e.g. in the pathintegral and hence, at least formally, quantization can be also performed without explicit restriction to the symplectic quotient. The formalism we have arrive at can be regarded as a presymplectic BV-AKSZ formalism or a version of BV-AKSZ formalism with constraints.

\subsection{The structure of the fiber and its symplectic quotient}

The space of (super)maps from $T[1] X$ to $\mathfrak{g}[1]$ can be locally represented as the space of (super)maps from $X$ to $M$, where $M$ is a space of (super)maps from $T_{x}[1] X$ to $\mathfrak{g}[1]$ for a given $x \in X$. It is clear that $M$ is finite-dimensional. If $\psi^{A}$ and $\theta^{\mu}$ are coordinates on $\mathfrak{g}[1]$ and $T_{x}[1] X$ respectively then a generic supermap is determined by a function $\psi^{A}(\theta)$ whose coefficients can be taken as coordinates on $M$. It is convenient to employ $\psi^{A}(\theta)$ as a generating function for coordinates on $M$. More precisely, we take as $\psi^{A}$ coordinates $\xi^{a}, \rho^{a b}$ on $\mathfrak{g}[1]$ and introduce coordinates on $M$ via

$$
\begin{aligned}
\xi^{a}(\theta) & =\xi^{a}+e_{\mu}^{a} \theta^{\mu}+\frac{1}{2} \stackrel{2}{\xi}_{\mu \nu}^{a} \theta^{\mu} \theta^{\nu}+\ldots+\frac{1}{n !} \xi_{\mu_{1} \ldots \mu_{n}}^{a} \theta^{\mu_{1}} \ldots \theta^{\mu_{n}} \\
\rho^{a b}(\theta) & =\rho^{a b}+\omega_{\mu}^{a b}(x) \theta^{\mu}+\frac{1}{2} \rho_{\mu \nu}^{a b} \theta^{\mu} \theta^{\nu}+\ldots+\frac{1}{n !} \rho_{\mu_{1} \ldots \mu_{n}} \theta^{\mu_{1}} \ldots \theta^{\mu_{n}}
\end{aligned}
$$

where by some abuse of notations we denote the $\theta$-independent component by $\xi^{a}$ and similarly for $\rho^{a b}$. The degree of the coordinates is determined by that of $\psi^{A}$ and $\theta^{\mu}$ so that $\operatorname{gh}(\stackrel{k}{\xi})=\operatorname{gh}(\stackrel{k}{\rho})=1-k$ and these coordinate functions provide a useful coordinate system on $M$. In the case at hand we chose $M$ to be the space of supermaps from $T_{x}[1] X$ to $\mathfrak{g}[1]$ satisfying the additional condition that the component $e_{\mu}^{a}$ entering $\xi^{a}(\theta)$ as $e_{\mu}^{a} \theta^{\mu}$ is required 
to be nondegenerate. Speaking geometrically, $M$ is a fiber bundle over $\operatorname{GL}(n, \mathbb{R})$. Note that coordinates $\stackrel{k}{\xi}$ and $\stackrel{k}{\rho}$ can be naturally interpreted (according to their index structure) as those parameterizing linear maps from $\wedge^{k} V$ to $V$ and $V \wedge V$ respectively (recall that $\left.T_{x} X \cong V\right)$ and whose ghost degree is shifted as explained above.

The presymplectic structure on $\mathfrak{g}[1]$ determines that on $M$ via

$$
\boldsymbol{\omega}^{M}=\int d^{n} \theta \boldsymbol{\omega}_{A B}^{\mathfrak{g}[1]}(\psi(\theta)) d \psi^{A}(\theta) \wedge d \psi^{B}(\theta),
$$

where $d \psi^{A}(\theta)$ is a generating function for basis differentials of coordinates on $M$. To analyse the structure of $\boldsymbol{\omega}^{M}$ it is convenient to consider a submanifold $M_{0} \subset M$ determined by

$$
\xi^{a}=0, \quad \stackrel{2}{\xi}_{\mu \nu}^{a}=0, \quad \ldots, \quad \stackrel{n}{\xi}_{\mu_{1} \ldots \mu_{n}}^{a}=0 .
$$

To understand the structure of the kernel of $\boldsymbol{\omega}^{M}$ it is instructive to consider $\boldsymbol{\omega}^{M}$ at a given point $p \subset M_{0}$. By changing the basis in $T_{x} X$ one can assume that $e_{\mu}^{a}=\delta_{\mu}^{a}$ to further simplify the analysis. In this basis the explicit expression for $\boldsymbol{\omega}^{M}$ at $p$ reads as

$$
\boldsymbol{\omega}_{p}^{M}=d e_{c}^{b} \wedge d \rho_{b}^{2}+d \xi^{b} \wedge d^{3} \rho_{b}+d \rho^{a b} \wedge d^{3} \xi_{a b}+d \omega_{c}^{a b} \wedge d \dot{\xi}_{a b}^{c}
$$

where new coordinate functions $\stackrel{2}{\rho}_{b}^{c}, \stackrel{3}{\rho} \rho_{b}, \stackrel{3}{\xi}_{a b}, \stackrel{2}{\xi}_{a b}^{c}$ parameterize

$$
\stackrel{2}{\rho_{b d} d}, \quad \stackrel{3}{\rho}_{b c d}^{c d}, \quad \stackrel{3}{\xi}_{a b c}^{c}, \quad \stackrel{2}{\xi}_{a b}^{c},
$$

respectively. More precisely, they are related via invertible linear map. The remaining components, which complete $\xi^{a}, e_{c}^{b}, \rho^{a b}, \omega_{c}^{a b}$ and functions (3.12) to a coordinate system on $M$ correspond to the kernel of $\boldsymbol{\omega}_{p}^{M}$. It is easy to see that the spectrum of coordinates along which $\boldsymbol{\omega}^{M}$ is nondegenerate is precisely that required for minimal BV formulation of GR. Let us stress that for the moment this is only established at $M_{0}$.

The crucial fact is that $M$ is a regular presymplectic manifold. To see this consider the following vector fields on $\mathfrak{g}[1]$ (here and below we restrict to $4 \mathrm{~d}$ to simplify the analysis):

$$
\begin{aligned}
X_{a}^{4} & =\xi^{(4)} \frac{\partial}{\partial \xi^{a}}, & X_{a b}^{3} & =\xi_{a}^{(3)} \frac{\partial}{\partial \xi^{b}}+\xi_{b}^{(3)} \frac{\partial}{\partial \xi^{a}} \\
Y_{a b}^{4} & =\xi^{(4)} \frac{\partial}{\partial \rho^{a b}}, & Y_{a b c}^{3} & =\xi_{a}^{(3)} \frac{\partial}{\partial \rho^{b c}}+\xi_{b}^{(3)} \frac{\partial}{\partial \rho^{a c}},
\end{aligned}
$$

where ... in the last expression denotes terms symmetryzing the expression in $a c$ and $b d$. Here $\xi_{a_{1} \ldots a_{4-k}}^{(k)}$ denote $\frac{1}{k !} \epsilon_{a_{1} \ldots a_{4-k} a_{4-k+1} \ldots a_{4}} \xi^{a_{4-k+1}} \ldots \xi^{a_{4}}$. It is easy to check that all these vector fields are in the kernel of $\boldsymbol{\omega}^{\mathfrak{g}[1]}$ and commute to one another.

By natural prolongation vector fields (3.13) on $\mathfrak{g}[1]$ determine the vector fields on $M$. Given a vector field $X$ on $\mathfrak{g}[1]$ the component expression for its prolongation $\widehat{X}$ can be obtained as follows:

$$
\widehat{X} \psi^{A}(\theta)=X^{A}(\psi(\theta)),
$$

where $X^{A}=X \psi^{A}$. Prolongation commutes with the commutator. In particular, prolongations of an involutive set of vector fields on $\mathfrak{g}[1]$ is again an involutive set. What is less 
trivial is that the distribution determined by an involutive set on $\mathfrak{g}[1]$ can be nonregular while its prolongation is regular. This happens because in our case $M$ is not the space of all maps but only of those whose $e_{\mu}^{a}$ component is invertible.

We have the following:

Lemma 3.1. The distribution determined by the prolongations of vector fields (3.13) coincides with the kernel distribution of $\boldsymbol{\omega}^{M}$ on $M$ and hence $\left(M, \boldsymbol{\omega}^{M}\right)$ is a regular presymplectic manifold.

Proof. First we show that on $M_{0}$ these vector fields exhaust the kernel of $\boldsymbol{\omega}^{M}$. This is easy to believe because the fields are linearly independent and their tensor structure precisely corresponds to the kernel of $\boldsymbol{\omega}^{M}$ at $M_{0}$, cf. (3.11). The proof is purely technical and is relegated to the appendix A.

Next, by construction vector fields $\widehat{X}, \widehat{Y}$ are in the kernel of $\boldsymbol{\omega}^{M}$ everywhere while at $M_{0}$ they exhaust the kernel. It follows they define the kernel everywhere. Indeed the dimension of the distribution determined by $\widehat{X}, \widehat{Y}$ can't drop when moving off $M_{0}$. Indeed, the dimension of the distribution is the rank of the matrix formed by the components of the defining vector fields and the rank can't decrease under infinitesimal deformations. Furthermore, the deformation in question is always infinitesimal because $M$ is a formal neighbourhood of $M_{0}$ ). At the same time the rank of $\boldsymbol{\omega}^{M}$ can not drop off $M_{0}$ by the same arguments so that it must be constant. ${ }^{2}$

The above arguments employs vector fields (3.13) that we explicitly gave only for the case of $n=4$. The proof can be extended to generic $n>4$ as follows. Observe that the linear space (over $\mathbb{R}$ ) of vector fields on $\mathfrak{g}[1]$ with $\rho$-independent coefficients is isomorphic to the tangent space $T_{p} M$ at $p \in M_{0}$. The image of a given vector field on $\mathfrak{g}[1]$ is determined by its prolongation to $M$ considered at $p$. In a similar way $T_{p}^{*} M$ is isomorphic to the space of 1 -forms on $\mathfrak{g}[1]$ with $\rho$-independent coefficients. Moreover, these isomorphisms are compatible with the map from vector fields (tangent vectors) to 1 -forms (resp. $T_{p}^{*} M$ ) determined by $\boldsymbol{\omega}^{\mathfrak{g}[1]}\left(\right.$ resp. $\left.\left.\boldsymbol{\omega}^{M}\right|_{p}\right)$. This implies that the kernel of $\left.\boldsymbol{\omega}^{M}\right|_{p}$ is determined by prolongations of the target space vector fields from the kernel of $\boldsymbol{\omega}^{\mathfrak{g}[1]}$ so that the above argument based on the rank of $\boldsymbol{\omega}^{M}$ completes the proof.

The regularity of $\left(M, \boldsymbol{\omega}^{M}\right)$ implies that there exists (at least locally) a symplectic quotient $N$ of $M$. In particular, by Frobenius theorem one can introduce a coordinate system $z^{i}, w^{\alpha}$ such that the vector fields $\widehat{X}, \widehat{Y}$ take the form $\frac{\partial}{\partial w^{\alpha}}$. The submanifold singled out by $w^{\alpha}=0$ and equipped with a pullback of $\boldsymbol{\omega}^{M}$ is isomorphic (as a symplectic manifold) to the symplectic quotient.

However, the Frobenius coordinates are not so easy to find explicitly. Nevertheless any submanifold transversal to the distribution determined by $\widehat{X}, \widehat{Y}$ and equipped with the induced symplectic structure is also isomorphic (as a symplectic manifold) to the symplectic quotient. Such transversal manifolds can be easily found using the following:

\footnotetext{
${ }^{2}$ Another way to see that is to fix the concrete values of $e_{\mu}^{a}, \omega_{\mu}^{a b}$ and consider $\boldsymbol{\omega}^{M}$ as function of the remaining coordinates. Then it has the form (3.11) plus terms proportional to the remaining coordinates of nonvanishing degree. But such terms can't decrease the rank. The same argument applies to the distribution determined by the vector fields.
} 
Lemma 3.2. Let $M$ be a graded presymplectic manifold and $M_{0} \subset M$ be its submanifold determined by equations $k_{r}=0$, where $\operatorname{gh}\left(k_{r}\right) \neq 0$. Let $z^{i}$, w $w^{\alpha}$ be local homogeneous (i.e. of definite degree) coordinates on $M$ such that $\boldsymbol{\omega}^{M}\left(\frac{\partial}{\partial w^{\alpha}}, \cdot\right)=0$ on $M_{0}, \frac{\partial}{\partial w^{\alpha}}$ determine a kernel of $\boldsymbol{\omega}^{M}$ at each point of $M_{0}$, and $\operatorname{gh}\left(w^{\alpha}\right) \neq 0$. Then (in general, locally defined) submanifold $\widetilde{N}$ determined by $w^{\alpha}=0$ is symplectic. Moreover, $\widetilde{N}$ is (locally) symplectomorphic to the symplectic quotient $N$ of $M$, provided $\boldsymbol{\omega}^{M}$ is regular.

Proof. The pullback $\boldsymbol{\omega}^{N}$ of $\boldsymbol{\omega}^{M}$ to $\tilde{N}$ is by construction nondegenerate at each point of $\widetilde{N}_{0}=\tilde{N} \cap M_{0}$. The standard considerations then ensure that $\omega^{N}$ can't degenerate off $\widetilde{N}_{0}$. This shows that $\widetilde{N}$ is symplectic.

Using the above Lemma it is not difficult to find a convenient choice of such functions $w^{\alpha}$. For instance, consider e.g.:

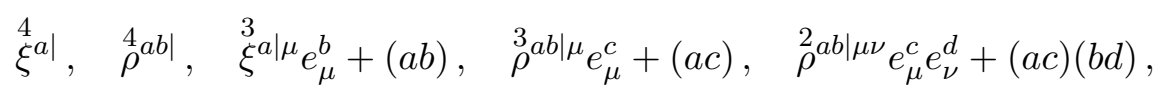

where e.g. $\stackrel{3}{\xi}^{a \mid \mu}$ stand for $\stackrel{3}{\xi}_{\nu \rho \sigma}^{a} \epsilon^{\mu \nu \rho \sigma}$. Because this set is manifestly $o(3,1)$ invariant it can be useful in practical computations though in this work we do not make use of these functions. Note the first 2 constraints originate from the target space in the sense that they can be represented as $\int d^{4} \theta \xi^{a}(\theta)$ and $\int d^{4} \theta \rho^{a b}(\theta)$. What is less trivial is that the last two can be replaced with those originating from the target space. More precisely, on $M_{0}$ they coincide with:

$$
\int d^{4} \theta \rho^{a b}(\theta) \xi^{c}(\theta)+(a c), \quad \int d^{4} \theta \rho^{a b}(\theta) \xi^{c}(\theta) \xi^{d}(\theta)+(a c)(b d) .
$$

This property could simplify implementation of these constraints in applications.

Given a regular presymplectic manifold $M$ we have the following:

Lemma 3.3. Let $Q$ be a homological vector field on $M$ satisfying $\mathfrak{i}_{Q} \boldsymbol{\omega}^{M}=d \mathcal{H}^{M}$ and $\boldsymbol{\omega}^{M}(Q, Q)=0$ for some function $\mathcal{H}^{M}$. It follows:

$$
\mathfrak{i}_{Q^{N}} \boldsymbol{\omega}^{N}=d \mathcal{H}^{N}, \quad \boldsymbol{\omega}^{N}\left(Q^{N}, Q^{N}\right)=0
$$

where $Q^{N}, \mathcal{H}^{N}$ and $\boldsymbol{\omega}^{N}$ are induced by $Q, \mathcal{H}^{M}$ and $\boldsymbol{\omega}^{M}$ respectively on the symplectic quotient $N$. If $N$ is identified with the submanifold $\widetilde{N} \subset M$ then $\mathcal{H}^{N}, \boldsymbol{\omega}^{N}$ can be identified with $\mathcal{H}^{M}, \boldsymbol{\omega}^{M}$ pulled back to $\widetilde{N}$.

Proof. The first part of the statement is standard and can be e.g. easily seen using special coordinates $z^{i}, w^{\alpha}$. That $\mathcal{H}^{N}, \boldsymbol{\omega}^{N}$ coincides with $\mathcal{H}^{M}, \boldsymbol{\omega}^{M}$ pulled back to $N$ is true because $\mathcal{H}^{M}, \boldsymbol{\omega}^{M}$ are constant along the kernel of $\boldsymbol{\omega}^{M}$. Indeed, from $\mathfrak{i}_{X} \boldsymbol{\omega}^{M}=0$ one finds $L_{X} \boldsymbol{\omega}^{M}=0$ and $X \mathcal{H}^{M}=\mathfrak{i}_{X} d \mathcal{H}^{M}=\mathfrak{i}_{X} \mathfrak{i}_{Q} \boldsymbol{\omega}^{M}=0$.

Note that the statement remains true if instead of regularity one requires $\widetilde{N} \subset M$ to be symplectic and such that $T_{p} M=T_{p} \widetilde{N} \oplus \operatorname{ker}\left(\boldsymbol{\omega}_{p}^{M}\right)$ for any $p \in \widetilde{N}$. In what follows we do not distinguish $N$ and $\widetilde{N}$ assuming that $N$ is either an abstract symplectic quotient or is identified as a suitable submanifold of $M$ 
Let us discuss the structure of $N$ and natural coordinates therein. First of all one observes that coordinates $\xi^{a}, \rho^{a b}, e_{\mu}^{a}, \omega_{\mu}^{a b}$ remain independent when restricted to $N$ and hence give a part of the coordinate system on $N$. The remaining coordinates are of negative ghost degree so that there is an invariantly defined submanifold $N_{01} \subset N$ obtained by setting them to zero.

Lemma 3.4. $N_{01}$ is a Lagrangian submanifold of $N . N$ can be identified as $T^{*}[-1] N_{01}$.

Proof. Using standard coordinates on $M$ one finds that

$$
\boldsymbol{\omega}^{M}\left(\frac{\partial}{\partial \xi^{a}}, \frac{\partial}{\partial \rho^{b c}}\right)=\epsilon_{a b c d}\left(\stackrel{4}{\xi}_{\mu \nu \rho \sigma}^{a} \epsilon^{\mu \nu \rho \sigma}\right)
$$

so that indeed it vanishes when $\stackrel{4}{\xi}_{\mu \nu \rho \sigma}^{a}$ (which is of degree -3) is set to zero. In a similar way one finds that $\omega$ vanishes on all pairs of vectors tangent to $N_{01}$. Because $N_{01}$ is Lagrangian $N$ can locally be represented as $T^{*}[-1] N_{01}$. However, since $N$ is a graded manifold and coordinates on the fibers of $T^{*}[-1] N_{01}$ are of negative degree this holds globally.

Identification of $N$ as a $T^{*}[-1] N_{01}$ gives an adapted Darboux coordinates given by $\xi^{a}, \rho^{a b}, e_{\mu}^{a}, \omega_{\mu}^{a b}$ and their canonically conjuated antfields $\xi_{a}^{*}, \rho_{a b}^{*}, e_{a}^{* \mu}, \omega_{\mu}^{* a b}$ so that

$$
\omega^{N}=d \xi^{a} \wedge d \xi_{a}^{*}+d \rho^{a b} \wedge d \rho_{a b}^{*}+d e_{\mu}^{a} \wedge d e_{a}^{* \mu}+d \omega_{\mu}^{a b} \wedge d \omega_{\mu}^{* a b} .
$$

It is easy to see that promoting these variables to fields on $X$ gives a standard set of fields, ghosts and their conjugated antifields required for the BV formulation of the Cartan-Weyl GR, see e.g. [4] and also [29] for a recent discussion.

\subsection{BV from presymplectic AKSZ}

Let us now turn to the piece $\int \widehat{\sigma}^{*}(\chi)\left(d_{X}\right)$ of the AKSZ action (3.4) determined by the De Rham differential. To this end consider the jet-bundle $J_{X}(M)$ associated to $X \times M \rightarrow X$ and denote by $D_{\mu}$ the total derivatives on $J_{X}(M) . D_{\mu}$ are the canonical horizontal lifts of the basis vector fields $\frac{\partial}{\partial x^{\mu}}$ on the base $X$ and de Rham diiferential on $J_{X}(M)$ then decomposes as $d=\mathrm{d}_{\mathrm{h}}+\mathrm{d}_{\mathrm{v}}=d x^{\mu} D_{\mu}+\mathrm{d}_{\mathrm{v}}$. It is useful to identify vertical coordinates on $J_{X}(M)$ as coefficients of the generating functions $\psi^{A}(y, \theta)$ which are associated to the coordinate system $\psi^{A}$ on $\mathfrak{g}[1] . \psi^{A}(y, \theta)$ are formal power series in new auxiliary variables $y^{\mu}, \operatorname{gh}\left(y^{\mu}\right)=0$ and the coordinates $\theta^{\mu}, \operatorname{gh}\left(\theta^{\mu}\right)=1$, which are identified with coordinates on the fibers of $T[1] X$. In this representation it is clear that $J_{X}(M)$ can also be defined as a super jet-bundle associated to $T[1] X \times \mathfrak{g}[1] \rightarrow T[1] X$.

On $J_{X}(M)$ we define the homological vector field $D$ via its action on coordinates:

$$
D \psi^{A}(y, \theta)=\theta^{\mu} \frac{\partial}{\partial y^{\mu}} \psi^{A}(y, \theta), \quad D x^{\mu}=0 .
$$

Another useful representation for $D$ is as follows: $D \psi^{A}(y, \theta)=\theta^{\mu} D_{\mu} \psi^{A}(y, \theta)$.

The symplectic structure on $M$ defines a symplectic structure on $J_{X}(M)$ :

$$
\boldsymbol{\omega}^{J(M)}=\int d^{n} \theta\left(\boldsymbol{\omega}_{A B}^{\mathfrak{g}[1]}(\psi(\theta)) \mathrm{d}_{\mathrm{v}} \psi^{A}(\theta) \mathrm{d}_{\mathrm{v}} \psi^{B}(\theta)=\omega_{i j} \mathrm{~d}_{\mathrm{v}} z^{i} \mathrm{~d}_{\mathrm{v}} z^{j}\right.
$$


where in the last equality we used special coordinate system $z^{i}, w^{\alpha}$ on $M$, such that $\omega\left(\frac{\partial}{\partial w^{\alpha}}, \frac{\partial}{\partial w^{\beta}}\right)=0=\omega\left(\frac{\partial}{\partial w^{\alpha}}, \frac{\partial}{\partial z^{i}}\right)$ on $N$, and whose existence has been proved in the previous section. More precisely, $z^{i}, w^{\alpha}$ denote coordinates on $J_{X}(M)$ obtained by pulling back $z^{i}$ to $J_{X}(M)$.

Understood as a local function on $J_{X}(M)$ the integrand (over $X$ ) of the AKSZ masteraction (3.5) takes the following form:

$$
\begin{aligned}
L^{\mathrm{BV}} & =K+\mathcal{H}^{M}, \\
K & =\int d^{n} \theta \chi_{A}(\psi(\theta)) \theta^{\mu} D_{\mu} \psi^{A}(\theta), \quad \mathcal{H}^{M}=\int d^{n} \theta \mathcal{H}(\psi(\theta)),
\end{aligned}
$$

where $\chi_{A}$ are components of the presymplectic potential on $\mathfrak{g}[1]$ and we identify functions on $M$ and their pullbacks to $J_{X}(M)$.

In the jet-bundle terms the BRST differential $s$ (cf. (3.6)) is represented by a vertical evolutionary vector field $s$ :

$$
s=D+Q^{p r}
$$

where $Q^{p r}$ is the prolongation of $Q$ to $J_{X}(M)$ determined by $\left[Q^{p r}, D_{\mu}\right]=0$. Furthermore, one has the following relations

$$
\mathfrak{i}_{D} \boldsymbol{\omega}^{J(M)}=\mathrm{d}_{\mathrm{v}} K+D_{\mu}(\cdot)^{\mu}, \quad \mathfrak{i}_{D} \mathfrak{i}_{D} \boldsymbol{\omega}^{J(M)}=D_{\mu}(\cdot)^{\mu}
$$

as well as

$$
\mathfrak{i}_{D} \mathfrak{i}_{Q^{p r}} \boldsymbol{\omega}^{J(M)}=D_{\mu}(\cdot)^{\mu}
$$

which can be directly checked and amount to:

$$
\mathfrak{i}_{s} \mathfrak{i}_{s} \boldsymbol{\omega}^{J(M)}=\mathrm{d}_{\mathrm{v}} L^{\mathrm{BV}}+D_{\mu}(\cdot)^{\mu}, \quad \mathfrak{i}_{s} \mathfrak{i}_{s} \boldsymbol{\omega}^{J(M)}=D_{\mu}(\cdot)^{\mu},
$$

where we also took into account $\mathfrak{i}_{Q} \mathfrak{i}_{Q} \boldsymbol{\omega}^{M}=0$.

Let us now consider the jet sub-bundle $J_{X}(N) \subset J_{X}(M)$ determined by $w^{\alpha}=0$ and their prolongations. Note that $J_{X}(N)$ can be also seen as the jet-bundle associated to $X \times N \rightarrow X$. Restricting relations (3.26) to $J_{X}(N)$ one gets:

$$
\mathfrak{i}_{s^{N}} \boldsymbol{\omega}^{J(N)}=\mathrm{d}_{\mathrm{v}} L_{N}^{\mathrm{BV}}+D_{\mu}(\cdot)^{\mu}, \quad \mathfrak{i}_{s^{N}} \mathfrak{i}_{s^{N}} \boldsymbol{\omega}^{J(N)}=D_{\mu}(\cdot)^{\mu}
$$

where $s^{N}$ denotes the projection of $s$ from $J_{X}(M)$ to $J_{X}(N)$ (induced by the projection $M \rightarrow N)$ and $\boldsymbol{\omega}^{J(N)}, L_{N}^{\mathrm{BV}}$ denote the respective objects on $J_{X}(M)$ pulled back to $J_{X}(N)$. Here by some abuse of notations $D_{\mu}$ denotes a total derivative on either $J_{X}(M)$ of $J_{X}(N)$; this does not lead to confusions because $D_{\mu}$ are tangent to $J_{X}(N) \subset J_{X}(M)$. The above relations are obvious if one makes use of the special coordinate system on $J_{X}(M)$ induced by special coordinates $z^{i}, w^{\alpha}$ on $M$.

Because $N$ is symplectic we are dealing with the standard BV formulation so that the above can be rewritten as $\left(L_{N}^{\mathrm{BV}}, L_{N}^{\mathrm{BV}}\right)=D_{\mu}(\cdot)^{\mu}$, where $L_{N}^{\mathrm{BV}}$ is $L^{\mathrm{BV}}$ restricted to $J_{X}(N)$. To summarize: understood as a local function on $J_{X}(M)$ the AKSZ action (3.4) is precisely $L^{\mathrm{BV}}=K+\mathcal{H}^{M}$. The sub-bundle $J_{X}(N)$ obtained by factoring out the kernel of the 
symplectic structure on $M$ is the BV jet-bundle equipped with the BV symplectic structure. The restriction of $K+\mathcal{H}^{M}$ to $J_{X}(N)$ satisfies master-equation and hence determines the BV formulation of the frame-like gravity. This is indeed true because (i) $N$ is symplectic (ii) $L^{\mathrm{BV}}$ restricted to the body of $J_{X}(N)$ is just the Cartan-Weyl Lagrangian (iii) the terms linear in ghosts and antifields contain the complete set of gauge generators. Note that the resulting $\mathrm{BV}$ formulation on $J_{X}(N)$ does not depend on how exactly $N$ is realised as a submanifold of $M$.

Point (ii) is a reformulation of (2.8) in the language of jet-bundles. Indeed, as we have seen the body of $M$ coincides with the body of $N$. The same applies to the associated $J_{X}(M)$ and $J_{X}(N)$ and hence the restriction of $L^{\mathrm{BV}}$ to the body of $J_{X}(N)$ is just a jetspace version of (2.8). Another point that requires clarification is (iii) because this ensures that $L^{\mathrm{BV}}$ is a proper solution to the master equations. To see this let us spell out the explicit expression for $L^{\mathrm{BV}}$

$$
\begin{aligned}
L^{\mathrm{BV}}= & \int d^{n} \theta \epsilon_{a b c d}\left(\frac{1}{2} e^{c} e^{d}\left(d_{X} \omega^{a b}+\omega^{a}{ }_{e} \omega^{e b}\right)\right. \\
& \left.+\xi^{c} e^{d} \nabla^{2} \stackrel{a}{\rho}^{a b}+e^{c} e^{d^{2}} \stackrel{2}{\rho}_{e} \rho^{e b}+\stackrel{2}{\xi^{c}} \xi^{d}\left(d_{X} \omega^{a b}+\omega^{a}{ }_{e} \omega^{e b}\right)+\stackrel{2}{\xi^{c}} e^{d}(\nabla \rho)^{a b}+\ldots\right),
\end{aligned}
$$

where ... denote terms at least quadratic in ghost fields and $\nabla$ is a covariant differential determined by $\omega^{a b}$, e.g. $\nabla \rho^{a b}=d_{X} \rho^{a b}+\omega^{a}{ }_{e} \rho^{e b}+\omega^{b}{ }_{e} \rho^{a e}$, and for simplicity we set $n=4$ and $\Lambda=0$. According to the usual rules of BV formalism the first two terms in the second line encode gauge transformations of $e_{\mu}^{a}$ because $\stackrel{2}{\rho}_{\mu \nu}^{a b}$ parameterize antifields conjugate to $\xi^{a}$ while the last two explicit terms encode the gauge transformations of $\omega_{\mu}^{a b} \stackrel{2}{\xi}_{\mu \nu}^{a}$ parameterize antifields conjugate to $\left.\omega_{\mu}^{a b}\right)$. In so doing the ghost fields $\xi^{a}$ and $\rho^{a b}$ are to be identified with the gauge parameters (in a certain basis) of the diffeomorphisms and the local Lorentz transformations respectively.

However, it is difficult to explicitly compare $L^{\mathrm{BV}}$ with the standard expression [4] of the BV master action for Cartan-Weyl action because even the above terms involve $\stackrel{2}{\rho}_{\mu \nu}^{a b}$ which give an overcomplete set of coordinates in this sector and moreover the symplectic structure is not in the canonical Darboux form. Fortunately, in order to prove that $L^{\mathrm{BV}}$ is a correct BV master action all we need to demonstrate is that it is proper (i.e. all gauge generators are taken into account). It is enough to do so in quadratic approximation because nonlinear corrections can not bring extra degeneracy.

Let us analyze the linearization of the gauge symmetries encoded in (3.28) around the vacuum solution $e_{\mu}^{a}=\delta_{\mu}^{a}$ and $\omega_{\mu}^{a b}=0$. One finds:

$$
\delta \bar{\omega}_{c}^{a b}=\partial_{c} \rho^{a b}, \quad \delta \bar{e}_{b}^{a}=\rho_{b}^{a}+\partial_{b} \xi^{a},
$$

where $\bar{e}_{b}^{a}$ and $\bar{\omega}_{c}^{a b}$ are related to the linearized $e_{b}^{a}$ and $\omega_{c}^{a b}$ respectively through a linear invertible redefinition. The above are precisely the linearized gauge symmetries of the Cartan-Weyl action. Equivalently, these are the gauge symmetries of its quadratic approximation:

$$
\int_{X}\left(\bar{e}^{a} d_{X} \bar{\omega}^{b c} \mathcal{V}_{a b c}+\bar{\omega}^{a}{ }_{c} \bar{\omega}^{c b} \mathcal{V}_{a b}\right)
$$


which is also known as the frame-like action of massless spin-2 field. Thus we conclude that the master action $L^{\mathrm{BV}}$ is a proper solution to the master equations and hence provides a correct BV formulation of Einstein gravity.

\section{The origin of the target space structures}

As we have seen the target space $\left(\mathfrak{g}[1], q, \boldsymbol{\omega}^{\mathfrak{g}[1]}\right)$ in a natural way defines the complete BV formulation of general relativity. One may wonder how does this target space arise from the conventional formulation of gravity. This was mostly explained in [25] but the relation between the presymplectic 2-form and the BV antibracket was somewhat implicit. Here we give missing details.

Suppose we start with the BV-BRST complex of the metric gravity. The set of fields is given by the metric $g^{a b}$, diffeomorphism ghosts $\xi^{a}$ and their canonically conjugate antifields $g_{a b}^{*}$ and $\xi_{a}^{*}$. More geometrically, these variables are coordinates on the fiber $F$ of the underlying bundle $F \times X \rightarrow X$. The BV-BRST complex is given by local horizontal forms on the associated jet-bundle $J_{X}(F)$ equipped with the BRST differential and the horizontal differential $\mathrm{d}_{\mathrm{h}}$, for more details see e.g. [4].

In the jet-bundle approach the standard symplectic structure of BV formulation is given by

$$
\boldsymbol{\omega}^{s B V}=(d x)^{n}\left(\mathrm{~d}_{\mathrm{v}} g^{a b} \wedge \mathrm{d}_{\mathrm{v}} g_{a b}^{*}+\mathrm{d}_{\mathrm{v}} \xi^{a} \wedge \mathrm{d}_{\mathrm{v}} \xi_{a}^{*}\right) .
$$

It is of ghost degree -1 and horizontal form degree $n$. The BRST differential is an evolutionary vector field satisfying

$$
\mathfrak{i}_{s} \boldsymbol{\omega}^{s B V}=\mathrm{d}_{\mathrm{v}} L^{s B V}+\mathrm{d}_{\mathrm{h}}(\cdot)
$$

where $L^{s B V}$ is the integrand of the BV master action. It follows

$$
L_{s} \boldsymbol{\omega}^{s B V}=\mathrm{d}_{\mathrm{h}}(\cdot) \text {. }
$$

Moreover, at least locally one can complete $\boldsymbol{\omega}^{s B V}$ to a cocycle of the total BRST differential $\widetilde{s}=\mathrm{d}_{\mathrm{h}}+s:$

$$
\boldsymbol{\omega}^{t B V}=\boldsymbol{\omega}^{s B V}+\boldsymbol{\omega}_{n-1}^{s B V}+\ldots
$$

where $\boldsymbol{\omega}_{k}^{s B V}$ has horizontal form degree $k$ and ghost degree $n-1-k$.

Now we use the standard statement that for diffeomorphism-invariant systems, and gravity in particular, by changing variable one can bring the total BV-BRST complex to the form where $\widetilde{s}=d_{X}+s$ and then eliminate $x^{a}, d x^{a}$ as contractible pairs, see e.g. [4, 12, 42]. Mention also related discussions of BRST formulation of diffeomorphism invariance in various approaches $[43,44]$. In more geometrical terms, this means that the underlying BV jet-bundle seen as a $Q$-bundle over $T[1] X$ is locally-trivial (see [13] for more details).

Furthermore, by eliminating further contractible pairs the total BRST complex reduces $[4,12,42]$ to the minimal BRST complex ${ }^{3}$ of functions on the reduced ghost-extended

\footnotetext{
${ }^{3}$ Seen as a $Q$-manifold the BRST complex determines [18] $L_{\infty}$-algebra [45-47]. This relation is one to one if we restrict to formal $Q$-manifolds. In particular, this minimal BRST complex corresponds to the minimal model of the respective $L_{\infty}$-algebra.
} 
stationary surface $\mathcal{E}$ which can be coordinatized by

$$
\xi^{a}, \quad \rho^{a b}, \quad W_{c d}^{a b}, \quad W_{c d ; c_{1}}^{a b}, \ldots W_{c d ; c_{1} \ldots c_{l}}^{a b}, \ldots,
$$

where $\operatorname{gh}\left(\xi^{a}\right)=\operatorname{gh}\left(\rho^{a b}\right)=1, \operatorname{gh}\left(W_{\ldots}^{\cdots}\right)=0$ and variables $\ldots$ are totally traceless (i.e. any invariant contraction of indexes gives zero). ${ }^{4}$ Variables $\xi^{a}, \rho^{a b}$ originate from the diffeomorphism ghost and its antisymmetrized derivatives, while $W$-variables are related to the Weyl tensor and its algebraically-independent covariant derivatives restricted to the stationary surface.

Upon the elimination of contractible pairs, the $\widetilde{s}$-differential determines a differential $q$ on $\mathcal{E}$. Its complete explicit form in terms of intrinsic coordinates on $\mathcal{E}$ is not known except in the sector of ghost degree 1 variables:

$$
q \xi^{a}=\rho^{a}{ }_{c} \xi^{c}, \quad q \rho^{a b}=\rho^{a}{ }_{c} \rho^{c b}+\lambda \xi^{a} \xi^{b}+\xi^{c} \xi^{d} W_{c d}^{a b}, \quad \ldots
$$

Furthermore, $q W_{c d ; \ldots}^{a b}$ is again proportional to $W_{c d ; \ldots}^{a b}$, see e.g. [12, 48].

Local functions on $\mathcal{E}$ equipped with $q$ form the minimal BRST complex for Einstein gravity. ${ }^{5}$ Because the minimal complex is an equivalent reduction of the initial one the initial $\widetilde{s}$-cocycle $\boldsymbol{\omega}^{t B V}$ gives rise to the respective $q$-cocycle $\boldsymbol{\omega}^{\mathcal{E}}$ in the space of closed 2forms on $\mathcal{E}$ of total ghost degree $n-1$. In fact the general structure of such form is rather restricted and it can be $\operatorname{shown}^{6}$ that $\boldsymbol{\omega}^{\mathcal{E}}$ is precisely $\boldsymbol{\omega}^{\mathfrak{g}[1]}$ trivially extended from $\mathfrak{g}[1]$ to $\mathcal{E}$ (note that locally $\mathcal{E}$ is a product of $\mathfrak{g}[1]$ and the space of Weyl tensors $W_{c d . . .}^{a b}$ ).

Now one can consider a presymplectic AKSZ sigma model with the target space $\left(\mathcal{E}, q, \omega^{\mathcal{E}}\right)$ and try to reduce to the symplectic quotient. In the case at hand it is convenient to do it in two steps. In the first step one considers a distribution on $\mathcal{E}$ generated by vector fields $\frac{\partial}{\partial W_{c d ; . . .}^{a b}}$ which obviously belong to the kernel distribution. The quotient can be realized as the surface $W_{c d ; \ldots}^{a b}=0$ which is precisely $\mathfrak{g}[1]$ with the induced two form being $\boldsymbol{\omega}^{\mathfrak{g}[1]}$ and the $Q$-structure being the restriction of $q$ to the surface. In this way one systematically rederives the presymplectic AKSZ formulation of section 2 starting from the conventional BV-BRST formulation of gravity.

\footnotetext{
${ }^{4}$ Here we intentionally used the same notations as for coordinates on $\mathfrak{g}[1]$ to anticipate the relation between $\mathcal{E}$ and $\mathfrak{g}[1]$.

${ }^{5}$ Note that the supermanifold $\widetilde{\mathcal{E}}$ of this variables equipped with $Q$-structure encodes all the information of the initial gauge theory. Indeed, as was shown in [12], taking $\widetilde{\mathcal{E}}$ as a target space of the AKSZ sigmamodel gives an equivalent formulation of the initial system at the level of equations of motion so that the system is fully reconstructed. It's equations of motion and gauge symmetries are precisely those of the minimal unfolded formulation [49,50]. Note however, that the explicit form of $q$ and hence of the unfolded equations of motion is not known in the intrinsic terms of $\mathcal{E}$ but its existence, structure, and the implicit definition are easily arrived at starting from the standard BV-BRST complex. Analogous considerations apply to generic gauge theories though in contrast to gravity for linear theories formulations of this sort can be quite concise and explicit. See [12] and references therein for further details.

${ }^{6}$ To see this one can observe that elimination of the contractible pairs preserves the filtration by the order of derivatives. Together with the $q$-invariance and the ghost degree conditions this essentially fixes the form of the presymplectic structure. As an independent consistency check one can take the analogous presymplectic structure for linearized gravity computed in [26] and observe that the only component surviving the reduction is the linearization of $\boldsymbol{\omega}^{\mathfrak{g}[1]}$.
} 
It turns out that analogous considerations apply to a rather wide class of gauge theories, giving a more precise understanding of the supergeometrical structures underlying their frame-like Lagrangians and BV formulations. Various examples of such presymplectic AKSZ formulations can be found in [24].

\section{BFV phase space from presymplectic AKSZ}

Given an AKSZ model on a space-time manifold of the form $X=\Sigma \times \mathbb{R}^{1}$, where $\Sigma$ corresponds to spatial slice and $\mathbb{R}^{1}$ to the time-line it is known [20, 31, 51] (see also [38, 52] for related developments, generalizations and applications) that its BFV formulation is given by an AKSZ sigma model restricted to $T[1] \Sigma$. The change of the dimension of the source space shifts by 1 the degree of the AKSZ action and the symplectic structure so that indeed such BFV-AKSZ sigma models gives a correct BFV formulation of the underlying gauge system.

That the constructed BFV formulation is correct immediately follows from the AKSZ formulation [31] of the standard construction [53, 54] of the BV formulation from the BFV one. More precisely, the 1d AKSZ sigma model with the target space being the above BFV-AKSZ sigma model can be identified with the initial AKSZ sigma model. Indeed, the space of supermaps from $T[1] \mathbb{R}^{1}$ to $\operatorname{Smaps}(T[1] \Sigma, \mathfrak{g}[1])$ is naturally identified with $\operatorname{Smaps}\left(T[1]\left(\Sigma \times \mathbb{R}^{1}\right), \mathfrak{g}[1]\right)$ and it is easy to check that the respective AKSZ structures coincide.

Let us consider the BFV version of the above presymplectic AKSZ sigma-model, which is obtained by replacing $T[1] X$ with $T[1] \Sigma$. The construction of an analog $M_{H}=$ $\operatorname{Smaps}\left(T_{x}[1] \Sigma, \mathfrak{g}[1]\right)$ of the graded manifold $M$ is straightforward. Similarly to (3.8) we introduce coordinates on $M_{H}$ as coeffcients of the generating functions $\xi^{a}(\theta)$ and $\rho^{a b}(\theta)$ which in contrast to (3.8) now depend on $n-1$ coordinates $\theta^{i}$ on $T_{x}[1] \Sigma$. Another difference is that we distinguish the spatial $e_{j}^{k}, k, j=1, \ldots, n-1$ and temporal components $e_{j}^{0}$ of the coordinates $e_{i}^{a}$ entering $\xi^{a}(\theta)$ as $e_{i}^{a}(x) \theta^{i}$ and restrict ourselves to (super)maps such that $e_{j}^{k}$ is invertible. Note that now the presymplectic structure $\boldsymbol{\omega}^{M_{H}}$ and "Hamiltonian" $\mathcal{H}_{H}$ have ghost degree 0 and 1 respectively, i.e. are shifted by 1 as compared to BV-AKSZ sigma model.

Just like $M, M_{H}$ is a graded presymplectic manifold. To see that it gives rise to a symplectic one let us apply Lemma 3.2 taking as $M_{H}^{0}$ a submanifold determined by $\xi^{a}=0, \stackrel{2}{\xi}_{i j}^{a}=0, \ldots, \stackrel{n-1}{\xi} \underset{i_{1} \ldots i_{n-1}}{a}=0$. Then fix a generic point of $M_{H}^{0}$ and adjust the basis in $T_{x}[1] \Sigma$ and $\mathfrak{g}[1]$ such that $e_{i}^{0}=0$ and $e_{i}^{a}=\delta_{i}^{a}$. At this point the presymplectic structure can be written as:

$$
\boldsymbol{\omega}_{p}^{M_{H}}=d e_{j}^{i} \wedge d \omega_{i}^{0 j}+d e_{j}^{0} \wedge d \omega^{j}+d \xi^{j} \wedge d^{2} \rho_{j}+d \xi^{0} \wedge d \stackrel{2}{\rho}+d \rho^{k 0} \wedge d \dot{\xi}_{k}+d \rho^{k j} \wedge d \dot{\xi}_{k j}^{0}
$$

where new coordinate functions $\omega^{j}, \stackrel{2}{\rho}{ }_{j}, \stackrel{2}{\rho}, \stackrel{2}{\xi}$ k are related to the following functions

$$
\omega_{k}^{k j}, \quad \stackrel{2}{\rho_{j k} k}, \quad \stackrel{2}{\rho_{j k}^{j k}}, \quad \stackrel{2}{\xi_{j k}^{k}}
$$


through some linear and invertible transformations. The coordinate functions entering (5.1) can be completed to the full coordinate system on $M_{H}$ by the following functions:

$$
\stackrel{3}{\xi^{a} \mid}, \quad \stackrel{3}{\rho} a b \mid, \quad \stackrel{2}{\xi}^{k \mid i} e_{i}^{j}+(i j), \quad \stackrel{2}{\rho}^{a k \mid i} e_{i}^{j}+(i j), \quad \omega^{i k \mid m n} e_{m}^{j} e_{n}^{l}+(i j)(k l),
$$

where e.g. $\stackrel{2}{\xi}^{k \mid i}$ stand for $\stackrel{2}{\xi}$ mn $\epsilon^{i m n}$ and similarly for $\stackrel{3}{\xi}{ }^{a \mid}, \stackrel{3}{\rho} a b|, \stackrel{2}{\rho} a k| i, \omega^{i k \mid m n}$. It is straightforward to see that these coordinates correspond to the kernel of $\boldsymbol{\omega}^{M_{H}}$ so that Lemma 3.2 implies that a submanifold $N_{H}$ where these coordinates vanish is symplectic. Moreover, the spectrum of the coordinates along which (5.1) is nondegenerate precisely corresponds to the coordinates of the BFV phase space for Cartan-Weyl formulation of gravity. More precisely, the BFV phase space we have arrived at is the one discussed recently in [28]. Note that there exist alternative (but equivalent) BFV formulations which are related through elimination of BFV analogs [19,20] of the conventional generalized auxiliary fields [55].

Let us consider the body $M_{H}^{\text {body }} \subset M_{H}$, obtained by setting to zero all the coordinates of nonvanishing degree. In contrast to the BV case the restriction of symplectic structure to the body is nonvanishing. Indeed, setting all the nonvanishing degree coordinates to zero the resulting presymplectic form takes the following form:

$$
\boldsymbol{\omega}^{M_{H}^{\text {body }}}=e_{i}^{a} d e_{j}^{b} \wedge d \omega_{k}^{c d} \epsilon^{i j k} \epsilon_{a b c d} .
$$

This 2-form is degenerate and moreover is a regular presymplectic one. Indeed, consider a generic point of $M_{H}^{\text {body }}$ and chose a basis such that $e_{i}^{0}=0$ and $e_{j}^{i}=\delta_{j}^{i}$. In this basis the 2-form coincides with (5.1), restricted to the tangent space to $M_{H}^{\text {body }}$ and hence its rank is constant. Furthermore, as we are going to see the symplectic structure induced by (5.4) on the symplectic quotient of $M_{H}^{\text {body }}$ gives rise to the phase space symplectic structure. More precisely, the phase space symplectic structure arises as that induced by (5.4) on the space of maps from $\Sigma$ to the symplectic quotient of $M_{H}^{\text {body }}{ }^{7}$

Note that the quotient can be described more invariantly [28]. Namely, consider the following equivalence relation on the space with coordinates $\omega_{i}^{a b}$ :

$$
\omega_{i}^{a b} \sim \omega_{i}^{a b}+v_{i}^{a b}, \quad v_{i}^{a b} e_{j}^{c} \epsilon^{i j k} \epsilon_{a b c d}=0
$$

Using the adapted basis it is easy to check that this equivalence relation precisely removes the trace-free component of $\omega_{i}^{j l}$ leading to the restriction of (5.1) to the body of $N_{H}$.

Having chosen symplectic $N_{H} \subset M_{H}$ let us consider the BFV phase space $J_{\Sigma}\left(N_{H}\right)$. It is equipped with the induced symplectic structure and the local functional $S^{B F V}$ obtained by restricting AKSZ BFV charge from $J_{\Sigma}\left(M_{H}\right)$ to $J_{\Sigma}\left(N_{H}\right)$. The restrictions of ghost degree 1 coordinates $\xi^{a}$ and $\rho^{a b}$ to $N_{H}$ remain independent and are to be interpreted as ghost variables. The terms in $S^{B F V}$ linear in $\xi^{a}$ and $\rho^{a b}$ read respectively as:

$$
\int_{\Sigma} \xi^{a} \epsilon_{a b c d} e^{b}\left(d_{\Sigma} \omega^{c d}+\omega^{c}{ }_{e} \omega^{e d}\right), \quad \int_{\Sigma} \rho^{a b} \epsilon_{a b c d} e^{c}\left(d_{\Sigma} e^{d}+\omega^{d}{ }_{f} e^{f}\right) .
$$

\footnotetext{
${ }^{7}$ Another way, employed recently in [28], to arrive at this presymplectic structure is to start with CartanWeyl action and find the presymplectic current (see e.g. [25, 56-58]) that it defines on the stationary surface. The present derivation of this presymplectic structure from that on $\mathfrak{g}[1]$ was somewhat implicitly already in [24].
} 
The coefficients are precisely the 1-st class constraints encoded in the Cartan-Weyl action. It follows $S^{B F V}$ have the structure similar to that of the proper BRST charge of the theory in question. What does not follow from the above considerations is that $S^{B F V}$ satisfies master equation on $J_{\Sigma}\left(N_{H}\right)$. If $M_{H}$ were a regular presymplectic manifold this would follow just like in BV case. However, the presymplectic structure on $M_{H}$ is in fact not regular and the detailed analysis of the presymplectic BFV-AKSZ formulation of gravity will be performed elsewhere.

Here we only give a simple argument showing the non-regularity: suppose the contrary, i.e. that $M_{H}$ is regular. Then it can be locally represented as $N_{H} \times W$, where $N_{H}$ is the symplectic manifold introduced above (symplectic quotient of $M_{H}$ ) and $W$ carries vanishing presymplectic structure. At the same time $M$ can be represented as $\operatorname{Smaps}\left(\mathbb{R}^{1}[1], M_{H}\right)$ and hence also factorises into $\operatorname{Smaps}\left(\mathbb{R}^{1}[1], N_{H}\right) \times \operatorname{Smaps}\left(\mathbb{R}^{1}[1], W\right)$, where the second factor has vanishing presymplectic structure while the first one must coincide with $N$ (the symplectic quotient of $M)$. In its turn $\operatorname{Smaps}\left(\mathbb{R}^{1}[1], N_{H}\right)$ can be identified with $T[-1] N_{H}$ so that one arrives at contradiction because even the spectrum of coordinates of $M$ is different from that of $T[-1] N_{H}$ (for instance in the sector of degree 0 coordinates on $N$ there are all components of $\omega_{k}^{i j}$ (recall that $i, j, k=1, \ldots, n-1$ ) while on $T[-1] N_{H}$ we do not find those which are in the kernel of the trace, i.e. components satisfying $\omega_{j}^{i j}=0$.

Let us finally comment on the relation between BV and BFV formulations arising from the presymplectic AKSZ. As we have just seen $M$ does not coincide with $T[-1] M_{H}$ and hence these BV and BFV formulations can not be related through the usual construction $[31,53,54]$. More precisely, the BV formulation obtained from the above BFV in this way is a certain equivalent reduction of the standard one. Indeed, as we have just seen not all components of the Lorentz connection are independent coordinates on $M_{H}$ and the same applies to $T[-1] M_{H}$ whose coordinates are the fields of the associated BV formulation. At the same time in the standard BV formulation for the Cartan-Weyl action all the components of the Lorentz connection are independent fields. This subtlety seems to be directly related to the discrepancy observed and investigated recently in [27, 28]. Despite this subtlety, the respective presymplectic BV and BFV AKSZ sigma models described in this work are obviously related via a straightforward presymplectic extension of the $1 \mathrm{~d}$ AKSZ construction of [31].

\section{Conclusions}

By concentrating on the example of general relativity we have demonstrated that presymplectic AKSZ-type sigma models naturally encode BV as well as BFV formulations in a rather concise and geometrical way. In so doing we have uncovered an interesting supergeometrical structures underlying the BV formulation of the frame-like gravity. This makes more explicit the deep relation between the underlying Cartan geometry and the BV formulation of gravity.

The present construction can be regarded as the BV extension of the so-called intrinsic Lagrangians [25], which are natural 1st order Lagrangian defined in terms of the equation manifold (stationary surface of the theory) equipped with the horizontal differential and the presymplectic current. 
It is important to stress that for various applications the presymplectic AKSZ formulation can be used in place of the conventional AKSZ. For instance, the formal path integral can be written just in terms of the presymplectic AKSZ action. The only difference is that some additional gauge-fixing conditions taking care of the kernel of the presymplectic 2-form are to be implemented in the path integral. Analogous remark applies to the presymplectic generalization of the generic (not necessarily AKSZ) BV fomalism.

Possible further developments include the extension of the present considerations to general local gauge theories including those which are not diffeomorphism-invariant. This can be naturally done using the language of gauge PDEs [13] equipped with the compatible presymplectic structures. There also remains to investigate further the BFV interpretation of the presymplectic AKSZ formulation of gravity as well as the presymplectic AKSZ version of the relation between its BV and BFV descriptions.

An attractive feature of the AKSZ formalism is that it makes manifest the relation between the bulk theory and the theory induced on the boundary. This feature already manifest itself in that Hamiltonian formulation (seen as a boundary theory induced on the surface of the initial data) is obtained by simply pulling back the AKSZ model to the boundary $[19,20,38,51,59]$. Applications to more general situations including holographic relations can be found in [27, 60-64]. Let us also mention recent works [65, 66], where the presymplectic structure (also known as the presymplectic current, see e.g. [56-58]) induced on the space of solutions to frame-like gravity is employed in the study of its boundary structure.

\section{Acknowledgments}

M.G. acknowledges discussions with G. Barnich and V. Gritsaenko. He is also grateful to A. Cattaneo, G. Canepa, M. Schiavina for the discussions and for attracting attention to their recent related works $[27,28]$. The work of M.G. was supported in part by the Russian Science Foundation grant 18-72-10123. The research of A.K. was supported by the grant no. 18-00496S of the Czech Science Foundation.

\section{A The structure of the kernel}

Here we show that prolongations of the vector fields (3.13) determine the kernel of $\boldsymbol{\omega}^{M}$ at generic $p \in M_{0}$. To begin with it is easy to see that $\widehat{X}_{a}^{4}$ and $\widehat{Y}_{a b}^{4}$ exhaust the kernel of $\boldsymbol{\omega}^{M}$ in the sector of $\stackrel{4}{\xi}_{\mu \nu \rho \sigma}^{a}$ and $\stackrel{4}{\xi}_{\mu \nu \rho \sigma}^{a b}$.

Consider then $\widehat{X}_{a b}^{3}$. At $p \in M_{0}$ one has:

$$
\widehat{X}_{a b}^{3}=\epsilon_{a c d f} e_{\mu}^{c} e_{\nu}^{d} e_{\rho}^{f} \frac{\partial}{\partial \xi_{\mu \nu \rho}^{b}}+(a b)
$$

where $(a b)$ denote terms symmetrizing the expression in $a$ and $b$. Using the basis where $e_{\mu}^{a} \delta_{\mu}^{a}$ and introducing notation $\stackrel{3}{\xi}^{a \mid e}$ for $\epsilon^{e f c d} \stackrel{3}{\xi}_{f c d}^{a}$ one finds:

$$
\widehat{X}_{a b}^{3}=\frac{\partial}{\partial^{3} \xi^{a \mid b}}+(a b) .
$$


Using symmetric and antisymmetric components $\widehat{\xi}_{S}^{a b}$ and $\widehat{\xi}_{A}^{a b}$ as new coordinates it is easy to see that $\widehat{X}_{a b}^{3}=\frac{\partial}{\partial \hat{\xi}_{S}^{a b}}$ while $\boldsymbol{\omega}^{M}$ is nondegenerate on $\frac{\partial}{\partial \hat{\xi}_{A}^{a b}}$. These later coordinates parameterize the antifields conjugated to $\rho^{a b}$, cf. (3.11).

Now we turn to $\widehat{Y}_{a b c d}^{2}$. As before restricting to a generic point of $M_{0}$ and using a basis where $e_{\mu}^{a}=\delta_{\mu}^{a}$ introduce new coordinates replacing $\rho$ :

$$
\bar{\rho}^{a b c d}=\epsilon^{a b \mu \nu} \rho_{\mu \nu}^{2}
$$

This is an invertible change of coordinates. In the new coordinates:

$$
\widehat{Y}_{a b c d}^{2}=\frac{\partial}{\partial \bar{\rho}^{a b c d}}+(a c)(b d)
$$

where $(a c)(b d)$ denote 3 terms symmetrizing $a c$ and $b d$.

In terms of $\bar{\rho}$ the trace $\stackrel{2}{\rho}_{c b}^{a b}$ can be expressed in terms of $\epsilon_{a b c d} \bar{\rho}^{\text {ebcd }}$ and one gets

$$
\widehat{Y}_{a b c d}^{2}\left(\epsilon_{\mu \nu \rho \sigma} \bar{\rho}^{\alpha \nu \rho \sigma}\right)
$$

so that the trace is not in the kernel of the presymplectic structure. The complementary component is described by $\rho$ satisfying $\epsilon_{a b c d} \bar{\rho}^{e b c d}=0$, i.e. having the symmetry type associated to rectangular YT. But this is precisely the tensor structure of $\widehat{Y}_{a b c d}^{2}$. Using new coordinate system $\hat{\rho}^{a b c d}, \widehat{\rho}^{a b}$, where $\widehat{\rho}^{a b c d}$ is the component of $\bar{\rho}^{a b c d}$ that have symmetry structure described by the rectangular YT while $\widehat{\rho}^{a b}$ parameterize the trace, one finds:

$$
\widehat{Y}_{a b c d}^{2}=\frac{\partial}{\partial \widehat{\rho}^{a b c d}}+(a c)(b d)
$$

so that indeed $\frac{\partial}{\partial \widehat{\rho}^{a b c d}}$ are in the kernel of the symplectic form. It is easy to check that among $\frac{\partial}{\partial \widehat{\rho}^{a b}}$ there are no zero vectors of the presymplectic form. In fact $\widehat{\rho}^{a b}$ parameterize antifields associated to $e_{\mu}^{a}$.

The remaining fields are $\widehat{Y}_{a b c}^{3}$. Introduce new parameterization of $\stackrel{3}{\rho}_{\mu \nu \rho}^{a b}$ in terms of $\bar{\rho}^{c \mid a b}$ proportional to $\epsilon^{c \mu \nu \rho} \stackrel{3}{\rho}_{\mu \nu \rho}^{a b}$ so that

$$
\widehat{Y}_{a b c}^{3}=\frac{\partial}{\partial \bar{\rho}^{a \mid b c}}+(a b)
$$

$\widehat{Y}_{a b c}^{3}$ contains two irreducible components: $\epsilon_{a b c d} \bar{\rho}^{a \mid b c}$ and $\bar{\rho}^{a \mid b c}+(a b)$. The first is precisely the double trace $\stackrel{3}{\rho}_{a b d}^{a b}$ which satisfies $Y_{a b c}^{3}{ }^{3} \rho_{e f d}^{e f}=0$. The second ones give rise to $Y_{a b c}^{3}$. Presymplectic form $\boldsymbol{\omega}^{M}$ is nondegenerate along $\stackrel{3}{\rho}_{a b d}^{a b}$.

Open Access. This article is distributed under the terms of the Creative Commons Attribution License (CC-BY 4.0), which permits any use, distribution and reproduction in any medium, provided the original author(s) and source are credited. 


\section{References}

[1] I.A. Batalin and G.A. Vilkovisky, Gauge algebra and quantization, Phys. Lett. B 102 (1981) 27 [INSPIRE].

[2] I.A. Batalin and G.a. Vilkovisky, Feynman rules for reducible gauge theories, Phys. Lett. B 120 (1983) 166 [INSPIRE].

[3] G. Barnich, F. Brandt and M. Henneaux, Local BRST cohomology in the antifield formalism. 1. General theorems, Commun. Math. Phys. 174 (1995) 57 [hep-th/9405109] [InSPIRE].

[4] G. Barnich, F. Brandt and M. Henneaux, Local BRST cohomology in Einstein Yang-Mills theory, Nucl. Phys. B 455 (1995) 357 [hep-th/9505173] [InSPIRE].

[5] G. Barnich, F. Brandt and M. Henneaux, Local BRST cohomology in gauge theories, Phys. Rept. 338 (2000) 439 [hep-th/0002245] [INSPIRE].

[6] R. Stora, Algebraic structure and topological origin of anomalies, seminar given at Cargese Summer Institute: Progress in Gauge Field Theory, September 1-15, Cargese, France (1983).

[7] M. Dubois-Violette, M. Talon and C.M. Viallet, BRS algebras: analysis of the consistency equations in gauge theory, Commun. Math. Phys. 102 (1985) 105 [INSPIRE].

[8] O. Piguet and S.P. Sorella, Algebraic renormalization: perturbative renormalization, symmetries and anomalies, Lecture Notes in Physics voluem 28, Springer, Germany (1995) [INSPIRE].

[9] G. Barnich, M. Grigoriev, A. Semikhatov and I. Tipunin, Parent field theory and unfolding in BRST first-quantized terms, Commun. Math. Phys. 260 (2005) 147 [hep-th/0406192] [INSPIRE].

[10] S.L. Lyakhovich and A.A. Sharapov, BRST theory without Hamiltonian and Lagrangian, JHEP 03 (2005) 011 [hep-th/0411247] [INSPIRE].

[11] P.O. Kazinski, S.L. Lyakhovich and A.A. Sharapov, Lagrange structure and quantization, JHEP 07 (2005) 076 [hep-th/0506093] [INSPIRE].

[12] G. Barnich and M. Grigoriev, First order parent formulation for generic gauge field theories, JHEP 01 (2011) 122 [arXiv: 1009.0190] [INSPIRE].

[13] M. Grigoriev and A. Kotov, Gauge PDE and AKSZ-type Sigma models, Fortsch. Phys. 67 (2019) 1910007 [arXiv: 1903.02820] [InSPIRE].

[14] G. Barnich and M. Grigoriev, BRST extension of the non-linear unfolded formalism, in the proceedings of the International School/Seminar on Quantum Field Theory, Supersymmetry, High Spin Fields, Gravity, March 20-26, Tomsk, Russia (2005) [hep-th/0504119] [InSPIRE].

[15] A. M. Vinogradov, Geometry of nonlinear differential equations, J. Sov. Math. 17 (1981) 1624.

[16] A. Vinogradov, Cohomological analysis of partial differential equations and secondary calculus, Translations of Mathematical Monographs volume 204, American Mathematical Society, U.S.A. (2001).

[17] J. Krasil'shchik and A. Verbovetsky, Geometry of jet spaces and integrable systems, J. Geom. Phys. 61 (2011) 1633 [arXiv:1002.0077] [InSPIRE].

[18] M. Alexandrov, A. Schwarz, O. Zaboronsky and M. Kontsevich, The Geometry of the master equation and topological quantum field theory, Int. J. Mod. Phys. A 12 (1997) 1405 [hep-th/9502010] [INSPIRE]. 
[19] M. Grigoriev, Parent formulation at the Lagrangian level, JHEP 07 (2011) 061 [arXiv: 1012.1903] [INSPIRE].

[20] M. Grigoriev, Parent formulations, frame-like Lagrangians, and generalized auxiliary fields, JHEP 12 (2012) 048 [arXiv: 1204.1793] [InSPIRE].

[21] I.A. Batalin and E.s. Fradkin, A generalized canonical formalism and quantization of reducible gauge theories, Phys. Lett. B 122 (1983) 157 [INSPIRE].

[22] I.A. Batalin and G.A. Vilkovisky, Relativistic $S$ matrix of dynamical systems with boson and fermion constraints, Phys. Lett. B 69 (1977) 309 [INSPIRE].

[23] H. Weyl, Elektron und gravitation. I, Z. Physik A 56 (1929) 330.

[24] K.B. Alkalaev and M. Grigoriev, Frame-like Lagrangians and presymplectic AKSZ-type sigma models, Int. J. Mod. Phys. A 29 (2014) 1450103 [arXiv:1312.5296] [InSPIRE].

[25] M. Grigoriev, Presymplectic structures and intrinsic Lagrangians, arXiv:1606.07532 [INSPIRE].

[26] A.A. Sharapov, Variational tricomplex, global symmetries and conservation laws of gauge systems, SIGMA 12 (2016) 098 [arXiv:1607.01626] [INSPIRE].

[27] G. Canepa, A.S. Cattaneo and M. Schiavina, General relativity and the AKSZ construction, Commun. Math. Phys. 385 (2021) 1571 [arXiv:2006.13078] [InSPIRE].

[28] G. Canepa, A.S. Cattaneo and M. Schiavina, Boundary structure of general relativity in tetrad variables, arXiv:2001.11004 [INSPIRE].

[29] A.S. Cattaneo and M. Schiavina, BV-BFV approach to general relativity: Palatini-Cartan-Holst action, Adv. Theor. Math. Phys. 23 (2019) 1801 [arXiv:1707.06328] [INSPIRE].

[30] A.S. Cattaneo and G. Felder, A path integral approach to the Kontsevich quantization formula, Commun. Math. Phys. 212 (2000) 591 [math.qa/9902090].

[31] M.A. Grigoriev and P.H. Damgaard, Superfield BRST charge and the master action, Phys. Lett. B 474 (2000) 323 [hep-th/9911092] [INSPIRE].

[32] I. Batalin and R. Marnelius, Superfield algorithms for topological field theories, hep-th/0110140 [INSPIRE].

[33] J.-S. Park, Topological open p-branes, hep-th/0012141 [INSPIRE].

[34] D. Roytenberg, On the structure of graded symplectic supermanifolds and Courant algebroids, math/0203110 [INSPIRE].

[35] A. Kotov and T. Strobl, Characteristic classes associated to Q-bundles, Int. J. Geom. Meth. Mod. Phys. 12 (2014) 1550006 [arXiv:0711.4106] [InSPIRE].

[36] F. Bonechi, P. Mnev and M. Zabzine, Finite dimensional AKSZ-BV theories, Lett. Math. Phys. 94 (2010) 197 [arXiv:0903.0995] [INSPIRE].

[37] G. Barnich and M. Grigoriev, A Poincaré lemma for sigma models of AKSZ type, J. Geom. Phys. 61 (2011) 663 [arXiv:0905.0547] [InSPIRE].

[38] A.S. Cattaneo, P. Mnev and N. Reshetikhin, Classical BV theories on manifolds with boundary, Commun. Math. Phys. 332 (2014) 535 [arXiv:1201.0290] [INSPIRE].

[39] N. Boulanger, N. Colombo and P. Sundell, A minimal BV action for Vasiliev's four-dimensional higher spin gravity, JHEP 10 (2012) 043 [arXiv: 1205.3339] [INSPIRE].

[40] N. Ikeda, Lectures on AKSZ sigma models for physicists, arXiv:1204.3714 [INSPIRE]. 
[41] G. Bonavolontà and A. Kotov, Local BRST cohomology for AKSZ field theories: a global approach, in the proceedings of the Winter School in Mathematical Physics: Mathematical Aspects of Quantum Field Theory, January 29-February 3, Les Houches, France (2013) [arXiv:1310.0245] [INSPIRE].

[42] F. Brandt, Local BRST cohomology and covariance, Commun. Math. Phys. 190 (1997) 459 [hep-th/9604025] [INSPIRE].

[43] O. Moritsch, M. Schweda and S.P. Sorella, Algebraic structure of gravity with torsion, Class. Quant. Grav. 11 (1994) 1225 [hep-th/9310179] [INSPIRE].

[44] O. Piguet, Ghost equations and diffeomorphism invariant theories, Class. Quant. Grav. 17 (2000) 3799 [hep-th/0005011] [INSPIRE].

[45] J. Stasheff, On the homotopy associativity of H-spaces. I, Trans. Amer. Math. Soc. 108 (1963) 275.

[46] J. Stasheff, On the homotopy associativity of H-spaces. II, Trans. Amer. Math. Soc. 108 (1963) 293.

[47] T. Lada and J. Stasheff, Introduction to SH Lie algebras for physicists, Int. J. Theor. Phys. 32 (1993) 1087 [hep-th/9209099] [INSPIRE].

[48] F. Brandt, Gauge covariant algebras and local BRST cohomology, Contemp. Math. 219 (1998) 53 [hep-th/9711171] [INSPIRE].

[49] M.A. Vasiliev, Triangle identity and free differential algebra of massless higher spins, Nucl. Phys. B 324 (1989) 503 [inSPIRE].

[50] M.A. Vasiliev, Actions, charges and off-shell fields in the unfolded dynamics approach, Int. J. Geom. Meth. Mod. Phys. 3 (2006) 37 [hep-th/0504090] [InSPIRE].

[51] G. Barnich and M. Grigoriev, Hamiltonian BRST and Batalin-Vilkovisky formalisms for second quantization of gauge theories, Commun. Math. Phys. 254 (2005) 581 [hep-th/0310083] [InSPIRE].

[52] N. Ikeda and T. Strobl, BV and BFV for the H-twisted Poisson sigma model, Annales Henri Poincaré 22 (2021) 1267 [arXiv: 1912.13511] [InSPIRE].

[53] J.M.L. Fisch and M. Henneaux, Antibracket-antifield formalism for constrained Hamiltonian systems, Phys. Lett. B 226 (1989) 80 [INSPIRE].

[54] A. Dresse, J.M.L. Fisch, P. Gregoire and M. Henneaux, Equivalence of the Hamiltonian and Lagrangian path integrals for gauge theories, Nucl. Phys. B 354 (1991) 191 [INSPIRE].

[55] A. Dresse, P. Gregoire and M. Henneaux, Path integral equivalence between the extended and nonextended Hamiltonian formalisms, Phys. Lett. B 245 (1990) 192 [InSPIRE].

[56] C. Crnkovic and E. Witten, Covariant description of canonical formalism in geometrical theories, in Three hundred years of gravitation, S.W. Hawking and W. Israel eds., Cambridge University Press, Cambridge U.K. (1987).

[57] I. Khavkine, Presymplectic current and the inverse problem of the calculus of variations, J. Math. Phys. 54 (2012) 111502 [arXiv:1210.0802].

[58] A.A. Sharapov, On presymplectic structures for massless higher-spin fields, Eur. Phys. J. C 76 (2016) 305 [arXiv: 1602.06393] [INSPIRE].

[59] M.A. Grigoriev, A Lie group structure underlying the triplectic geometry, Phys. Lett. B 458 (1999) 499 [hep-th/9901046] [INSPIRE].

[60] X. Bekaert and M. Grigoriev, Notes on the ambient approach to boundary values of AdS gauge fields, J. Phys. A 46 (2013) 214008 [arXiv:1207.3439] [InSPIRE]. 
[61] X. Bekaert and M. Grigoriev, Higher order singletons, partially massless fields and their boundary values in the ambient approach, Nucl. Phys. B 876 (2013) 667 [arXiv:1305.0162] [INSPIRE].

[62] M. Grigoriev and E.D. Skvortsov, Type-B formal higher spin gravity, JHEP 05 (2018) 138 [arXiv: 1804.03196] [INSPIRE].

[63] P. Mnev, M. Schiavina and K. Wernli, Towards holography in the BV-BFV setting, Ann. Henri Poincaré 21 (2019) 993 [arXiv:1905.00952] [InSPIRE].

[64] K. Rejzner and M. Schiavina, Asymptotic symmetries in the BV-BFV formalism, Commun. Math. Phys. 385 (2021) 1083 [arXiv: 2002.09957] [INSPIRE].

[65] L. Freidel, M. Geiller and D. Pranzetti, Edge modes of gravity. Part III. Corner simplicity constraints, JHEP 01 (2021) 100 [arXiv:2007.12635] [INSPIRE].

[66] L. Freidel, M. Geiller and D. Pranzetti, Edge modes of gravity. Part II. Corner metric and Lorentz charges, JHEP 11 (2020) 027 [arXiv: 2007.03563] [INSPIRE]. 